\title{
NUEVOS DATOS SOBRE MYXOMYCETES PRESENTES EN LA PROVINCIA DE GRANADA (ESPAÑA)
}

\author{
R. GALAN, C. LADO \& A. ORTEGA
}

\begin{abstract}
RESUMEN: En el presente trabajo se aportan nuevos datos sctre el conocimiento de los Myxomycetes de Granada (sur de España), citándose catorce nuevas especies, entre las ciue se destacan: Comatricha alta Preusz, Diderma trevelyanii (Grev.) Fr., Diderma umbilicatum Pers., Didymium bahiense Gottsberger, Didymium difforme (Pers.) S.F. Gray, Didymium trachysporum G. Lister, Lamproderma scintillans (Berk. \& Br.) Morgan, Physarum contextum (Pers.) Pers., Physarum vernum Somm. y se amplía la corología de varias especies ya citadas en anteriores trabajos. Se comenta la distribución en la Peninsula Ibérica, confeccionándose mapas de distribución de dichos taxones basados en cuadricula UTM de $50 \mathrm{Km}$.
\end{abstract}

SUMMARY: This article shows a study on the Myxomycetes on the province of Granada (Spain). We have found fourteen new taxa for this province. Some of these are: Comatricha alta Preusz, Diderma trevelyanii (Grev.) Fr., Diderma umbilicatum Pers., Didymium bahiense Gottsberger, Didymium difforme (Pers.) S.F. Gray, Didymium trachysporum G. Lister, Lamproderma scintillans (Berk. E Br.) Morgan, Physarum contextum (Pers.) Pers., Physarum vernum Somm. Distribution maps are given. We also included new dates about the chorology another species.

\section{INTRODUCCION}

El estado de conocimiento sobre los Myxomycetes en la Península Ibérica es en la actualidad ciertamente importante, de modo que a las primeras citas de estos organismos, cuyo resumen histórico apuntan Ortega \& Calonge (1980), hay que sumar la gran labor monográfica de Almeida $(1964,1966,1972,1974-76,1977-79)$ en Portugal, Gracia et al. $(1977,1980,1981)$ y Gracia (1981) en el este y sureste español y Ladó \& al. $(1976,1978,1980,1981)$ principalmente en la región centro y norte de España, además de ciertos trabajos esporádicos de otros micólogos nacionales.

Con este trabajo y siguiendo la línea iniciada por Ortega \& Calonge (1980) y Ladó,Moreno, Ortega \& Calonge (1980), pretendemos dar a conocer la aparición de nuevas especies de Myxomycetes aún no catalogados en la provincia de Granada (con lo cual el número de estos asciende en la actualidad a 41 especies); dichas especies han sido ordenadas alfabéticamente y cada una de las mismas va acompañada de referencias que consideramos precisas con vista a su clara definición taxonómica y corológica. En una segunda lista se amplía la corología a nivel provincial de muchos otros táxones anteriormente recolectados.

Todo el material examinado se encuentra depositado en el herbario GDAC (Granada.Ciencias). 


\section{RESULTADOS}

Arcyria cinerea (Bull.) Pers.

Material estudiado: Alhama de Granada,1140 m, 30SVF19, madera de Quercus rotundifolia, Gil, 19.XI.1980, GDAC 11254.

Distribución: Navarra (Urries,1953); Madrid (Ladó \& Moreno,1976, 1980); (Checa \& al., 1982); Gerona, Barcelona (Gracia, 1977); Murcia (Gracia \& Llimona, 1980); País Vasco (Ladó \& Moreno, 1981); Barcelona, Gerona, Tarragona, Valencia (Gracia, 1981); Cádiz (Mitchell, com. pers.); Mafra,Lumiar, Queluz (Torrend, 1908); Cascais (Almeida, 1966); Próximo Rio Maior, Lisboa (Almeida, 1972); Lisboa (Almeida, 1974-76); Serta,Monte Gordo (Almeida, 1977-79); Guadalajara, Avila (Ladó, datos pers. ined.). Mapa 1.

Observaciones: Esta especie la podemos considerar como un típico ejemplo de taxon cosmopolita y ubiquista, ya que se encuentra distribuida por todo el mundo y sobre todo tipo de sustratos. En la Península Ibérica se encuentra igualmente distribuida por todo el territorio y en especial por el área mediterránea, en donde la encontramos citada desde Gerona hasta Cádiz. Nuestro material es una muestra escasa pero típica, con capilicio espinoso a dentado y un calículo ornamentado con tenues papilas. La coloración del esporangio, aunque algo variable en esta especie, se presenta con tonos grises blanque cinos típicos.

\section{Arcyria ferruginea Sauter}

Material estudiado: Alfaguara, $1400 \mathrm{~m}, 30$ SVG52, sobre madera de pino descortezada, Ortega, 26.V.1981, GDAC 11424. Ibidem, sobre madera de pino, Fernández et Cuadrado, 5.IV.1981, GDAC 11425. Ibidem, en madera muerta de pino, Ortega et Galán, 29.X.1981, GDAC 122 49 .

Distribución: Barcelona (Cuatrecasas, 1925) (Gracia, 1977,1981); Madrid (Ladó \& Moreno, 1976,1980) (García Manjón, 1978); Avila (Ladó datos pers. ined.); Alava (Cat. Mic. Alava, 1976); La Coruña,Pontevedra (Castro, com. pers.); Mafra,Tala-Belas,Lisboa (Almeida, 1964); Alcochete, Arrabida, Minas de Panasqueira (Almeida, 1977-79); Alfeite, Sintra (Torrend, 1908). Mapa 2.

Observaciones: Especie característica por su típica ornamentación del capilicio y por la presencia de esporas de gran tamaño (9-11 $\mu \mathrm{m}$ de diámetro) en relación con el resto de especies de este género. Se encuentra escasamente representada en nuestro país y en la mayoría de los casos se halla ligada a la madera de coníferas, más concretamente a Pinus. Creemos que su distribución en la Península es amplia pero se trata de una especie poco común.

Badhamia panicea (Fr.) Rost.

Material estudiado: Monachil, $780 \mathrm{~m}$, 30SVG51, corteza de Populus nigra, Isac et Galán, 17.V.1980, GDAC 11614.

Distribución: Barcelona (Gracia, 1977); Murcia (Gracia \& Llimona, 1980); Madrid (Ladó \& Moreno, 1980); Murcia,Alicante, Barcelona, Tarragona (Gracia, 1981); Toledo (Mitchell, com. pers.); Lumiar (Torrend, 1908). Mapa 3. 
Observaciones: Ampliamente distribuida por la Península y aunque si bien no se conocía en el sur,era de preveer su presencia en la provincia de Granada, como lo prueba el hecho de encontrarse frecuentemente citada a todo lo largo de la costa mediterránea peninsular.

\section{Comatricha alta Preusz}

Material estudiado: Alfaguara,1400 m, 30SVG52, madera descortezada de Pinus, Ortega, 26.V.1981, GDAC 11423.

Distribución: Murcia, Barcelona (Gracia, 1981); Cuenca (Ladó,datos pers. ined.). Mapa 4.

Observaciones: Especie compleja y poco frecuente, caracterizada por su forma oblongo-cilíndrica y por el capilicio largo y flexuoso que se suelta en la parte superior de la fructificación dejando la columnela libre,permaneciendo unido tan sólo en la parte inferior.

Esta especie ha sido considerada por algunos autores (Lister, 1911,1925) como una variedad de Comatricha nigra (Pers.) Schroet., por presentar algunos caracteres macroscópicos bastante similares, no obstante otros autores (Nannega-Bremenkamp, 1974) lo mantienen como un taxon independiente por considerar la antigua especie de Preusz con entidad propia y perfectamente diferenciable de Comatricha nigra en base a su capilicio que se suelta en la madurez, lo que no ocurre en Comatricha nigra. Particularmente somos partidarios de esta segun da opinión por lo que consideramos como tal dicho taxon.

En lo que se refiere a su corología, se conocen únicamente tres citas en la Península : Sierra de Espuña (Murcia), Montseny (Barcelona) y la Hoz de Beteta (Cuenca). Presenta cierta tendencia a desarrollarse en zonas montañosas con índice de humedad elevado y suele hacerlo en madera de coníferas.

Diderma trevelyanii (Grev.) Fr.

Material estudiado: Fuente Agrilla, carretera Seminario Sierra Nevada, $1400 \mathrm{~m}$, 30SVG61, tocón en descomposición posiblemente de Castanea sativa, Jiménez et Galán, 10.1II.1982, GDAC 12241.

Distribución: Avila (Ladó \& Moreno, 1978); Madrid (Ladó \& Moreno, 1980); Segovia (Ladó, datos pers. ined.). Mapa 5.

Observaciones: Sus fructificaciones sésiles o cortamente pediceladas, globosas, de color marrón, con peridio cartilaginoso y dehiscencia típica petaloide o estrellada, con columnela ausente o a menudo reducida a un pequeño gránulo en el centro del esporangio, además de su capilicio marrón claro, filamentoso y con nódulos más oscuros la diferencian de especies próximas. (Lam. 1: 1y2).

Se trata de una especie rara en la Península Ibérica, conociéndose únicamente en la zona centro. En Europa, su aparición queda relegada a los paises norte y centroeuropeos,localizándose en áreas montañosas y siendo desconocida en el área mediterránea. La explicación de la aparición de táxones de comportamiento ecológico y corológico similar al que tratamos, en la provincia de Granada, debe explicarse sin duda alguna dada la extremada variabilidad climática que posee esta provincia, fruto de su orografía, lo que hace que en zonas costeras se dé un clima termomediterráneo-semiárido, atendiendo a la terminología de Rivas-Martínez (1981) y en las zonas más elevadas de Sierra Nevada se alcance el piso crioromediterráneo pasan- 
do por grados intermedios como es el caso de la localidad que nos ocupa (meso-supramediterráneo, seco-subhúmedo).

\section{Diderma umbilicatum Pers.}

Material estudiado: Alhama de Granada, $1140 \mathrm{~m}, 30$ SVF19, ramitas muertas de Quercus rotundifolia, Ortega et Galán, 24.II.1982, GDAC 12252 .

Distribución: Madrid (Ladó \& Moreno, 1978,1981 como D. niveum); Albacete (Gracia et al., 1981) (Gracia, 1981); Gerona (Gracia, 1981). Mapa 6.

Observaciones: Especie compleja que ha estado considerada durante mucho tiempo como una variedad de Diderma radiatum (L.) Morgan, pero recientemente (Nannenga-Bremekamp, 1968) se ha reconsiderado la especie de Persoon en base a la naturaleza calcárea del peridio (sección Eudiderma) y no cartilaginosa (sección Leangium), como es el caso de D. radiatum, aparte de su coloración gris perla y su dehiscencia irregular. Su distribución en la Península es pobre, conociéndose únicamente de las provincias antes citadas y siempre ligada a localidades montañosas.

Didymium bahiense Gottsberg. emend. Nann.-Brem.

Material estudiado: Granada capital, $780 \mathrm{~m}, 30 \mathrm{SVG} 41$, en semillas descompuestas de Salvia sp. comercializadas con el nombre "semillas del árbol de la chía" y de origen presumiblemente mexicano, Galán, X.1981,GDAC 12244 .

Distribución: Gerona, Murcia, Albacete (Gracia, 1981). Mapa 7.

Observaciones: Esta especie, de reciente creación, se diferencia de otras próximas fundamentalmente por la presencia de pseudocolumnela (Lam. 1:5), además de sus esporas con dimensiones comprendidas entre (9-) 10-12 (-14) $\mu \mathrm{m}$ de diámetro y ornamentadas con verrugas de dos tamaños distintos que se disponen más o menos en grupos (Nannenga-Bremekamp, 1972). (Lam. 1:6).

En cuanto a su distribución en el mundo, se conocía únicamente de Brasil y Holanda. Sin embargo, por los datos aportados por Gracia (1981) y los nuestros propios, podemos deducir que este taxon se encuentra bien representado en la Península Ibérica si bien ocupando áreas térmicas y distribuyéndose por la España mediterránea.

\section{Didymium difforme (Pers.) S.F. Gray}

Material estudiado: Se encontró en similares condiciones a la especie anterior y conviviendo con la misma, GDAC 12245.

Distribución: Albacete (Gracia \& al., 1981); Albacete, Valencia, Alicante, Gerona, Barcelona, Lérida (Gracia, 1981); Toledo (Mitchell, com. pers.) Avila, Madrid, Segovia (Ladó, datos pers. ined.); Lisboa (Almeida, 1974-1976). Mapa 8.

Observaciones: Como podemos observar no es hasta 1981 (Gracia et al., 1981) cuando la presencia de esta especie se detecta en España, pero es a partir de ese año cuando aparecen numerosas recolectas de este taxon en la Península, distribuyéndose por una extensa área. 
En todos los casos pensamos que se halla ligada a zonas con marcado carácter térmico y también es de destacar que el sustrato sobre el que se desarrolla es en todos los casos restos de herbáceas y hojas, sustratos estos cuyos indices de acumulación de agua es escaso.

Didymium trachysporum G. Lister

Material estudiado: Granada capital,780 m,30SVG41. Tubérculo de boniato (Ipomoea batatas) en descomposición, Galán, 9.1II.1982, GDAC 12255 .

Distribución: Almería (Ladó \& Moreno,1976); Lisboa (Almeida, 1974-76). Mapa 9 .

Observaciones: Las fructificaciones en forma de esporangios sésiles o cortos plasmodiocarpos, blancos y pequeños $(0,2-0,4 \mathrm{~mm}$ de diámetrol,pulvinados, con escasos hilos de capilicio, esporas marrón oscuro y verrugas patentes de 9-10,5 $\mu \mathrm{m}$ de diámetro, caracterizan esta especie. (Lam.1:7).

Según Martin \& Alexopoulos (1969) es frecuente en el centro y oeste de Europa. Nuestra cita,junto a la de Ladó (1976) amplia el área de distribución de éste taxon a la Europa mediterránea.

Lamproderma scintillans (Berk. \& Br.) Morgan

Material estudiado: Carretera a Pinos del Valle,Güájares, 1000 $\mathrm{m}, 30 \mathrm{SVF}$ 48, sobre hojas en descomposición, Ortega \& Salvo, 22.XI.1979 GDAC 12243 .

Distribución: Gerona (Gracia, 1981); Setubal, Val de Rosal, Alfeite (Torrend, 1908); Lisboa (Almeida, 1974-76); Madrid, Segovia (Ladó, datos pers. ined.). Mapa 10.

Observaciones: Los escasos datos de que disponemos sobre esta especie en la Perínsula Ibérica, en lo que a su corología se refiere, pensamos que no quiere indicar su rareza, pues uno de nosotros(La dó, datos pers. ined.) ha podido constatar que es un taxon frecuente y ampliamente distribuido en las provincias de Madrid y Segovia, confirmando asi lo indicado por Kowalski (1970) quien al hablar de esta especie indica "Probably cosmopolitan, but more abundant in temperate lowland regions".

Physarum contextum (Pers.) Pers.

Material estudiado: Arroyo de Fardes, Alfaguara, $1440 \mathrm{~m}$, 30SVG 52, sobre ramas de herbáceas, Ortega \& Galán, 10.XII.1978, GDAC 12242 .

Distribución: Madrid (Caballero, 1928); Existe otra cita de Mas y Guindal (1945) pero se trata del mismo material de Caballero; Monchique, Benfica (Torrend, 1908). Mapa 11.

Observaciones: La cita de la provincia de Madrid dada por Caballero (1928) era, hasta el momento, el único dato de que disponíamos en España; sin embargo al no poder estudiar este material por no encontrarse depositado en ningún herbario por nosotros consultado y carecer de una, aunque escueta, descripción del material recolectado por este autor nos hace poner en duda dicha cita. En cualquier 
caso nuestro material viene a confirmar su presencia en España.

Por otra parte y según datos bibliográficos (Martin \& Alexopoulos, 1969), esta especie está considerada como de amplia distribución en toda Europa por lo que era de suponer su presencia en la Península Ibérica. (Lam. 1:8).

\section{Physarum vernum Somm.}

Material estudiado: Alhama de Granada, $1140 \mathrm{~m}, 30$ SVF19, sobre hojas de Quercus rotundifolia, Ortega \& Galán, 27.XI.1980, GDAC 11066.

Distribución: Barcelona (Gracia, 1977); Benfica (Torrend, 1908), cita esta que recoge Almeida (1974-76); Madrid (Ladó, datos pers. inéd.). Mapa 12.

Observaciones: Especie muy próxima a Physarum cinereum (Batsch) Pers., de la que se distingue por sus esporas más oscuras y de mayores dimensiones, por su peridio usualmente más calcáreo $y$ por la tendencia a fructificar en forma de plasmodiocarpos.

En cuanto a su distribución, nuestra cita viene a confirmar y ampliar la corología de esta especie en el área mediterránea.

\section{Trichia contorta (Ditmar) Rost. var. contorta}

Material estudiado: Güéjar Sierra, $1400 \mathrm{~m}$, 30SVG61, sobre ramas caidas de Quercus rotundifolia, Ortega \& Galán,18.XI.1980. GDAC 11070.

Distribución: Madrid (Ladó \& Moreno, 1976,1980); Murcia (Gracia \& Llimona, 1980)(Gracia,1981); Albacete (Gracia \& al., 1981) (Gracia, 1981); Avila,Guadalajara, Segovia (Ladó, datos pers. inéd.). Mapa 13.

Observaciones: Especie cosmopolita, ampliamente distribuida en la Península Ibérica pero desconocida hasta el momento en Andalucía.

\section{Trichia varia (Pers.) Pers.}

Material estudiado: Dehesa del Camarate, $1400 \mathrm{~m}, 30 \mathrm{SVG} 71$, en madera de Populus en descomposición, Ortega \& Galán, 20.XI.1980,GDAC 11255.

Distribución: Madrid (Benito Martinez \& Guinea, 1931) (Amo y Mora, 1870) (Mas y Guindal, 1945) (Ladó \& Moreno, 1980) (Checa \& al. 1982); Barcelona (Font-Quer, 1923) (Maire, 1933) (Gracia, 1977,1981); Alava (Cat. Mic. Alava, 1976); Albacete (Gracia \& al.,1981) (Gracia, 1981); Guipúzcoa (Ladó \& Moreno, 1981); Alicante,Gerona, Lérida (Gracia, 1981); Avila,Guadalajara, Segovia (Ladó, datos pers. inéd.); Lisboa (Almeida, 1964); Serta S. Bartolomeu do Messines, Monchique, Lisboa, Pinal do Rei (Almeida, 1977-79); Arrabida, Mata do Vidal (Almeida, 1972); Lumiar, Sintra, Mafra, Coimbra, S. Fiel (Torrend,1908). Mapa 14.

Observaciones: Se trata de una especie cosmopolita y que se encuentra ampliamente distribuida en toda la Península pero al igual que el taxon precedente era aún desconocida en Andalucía. 


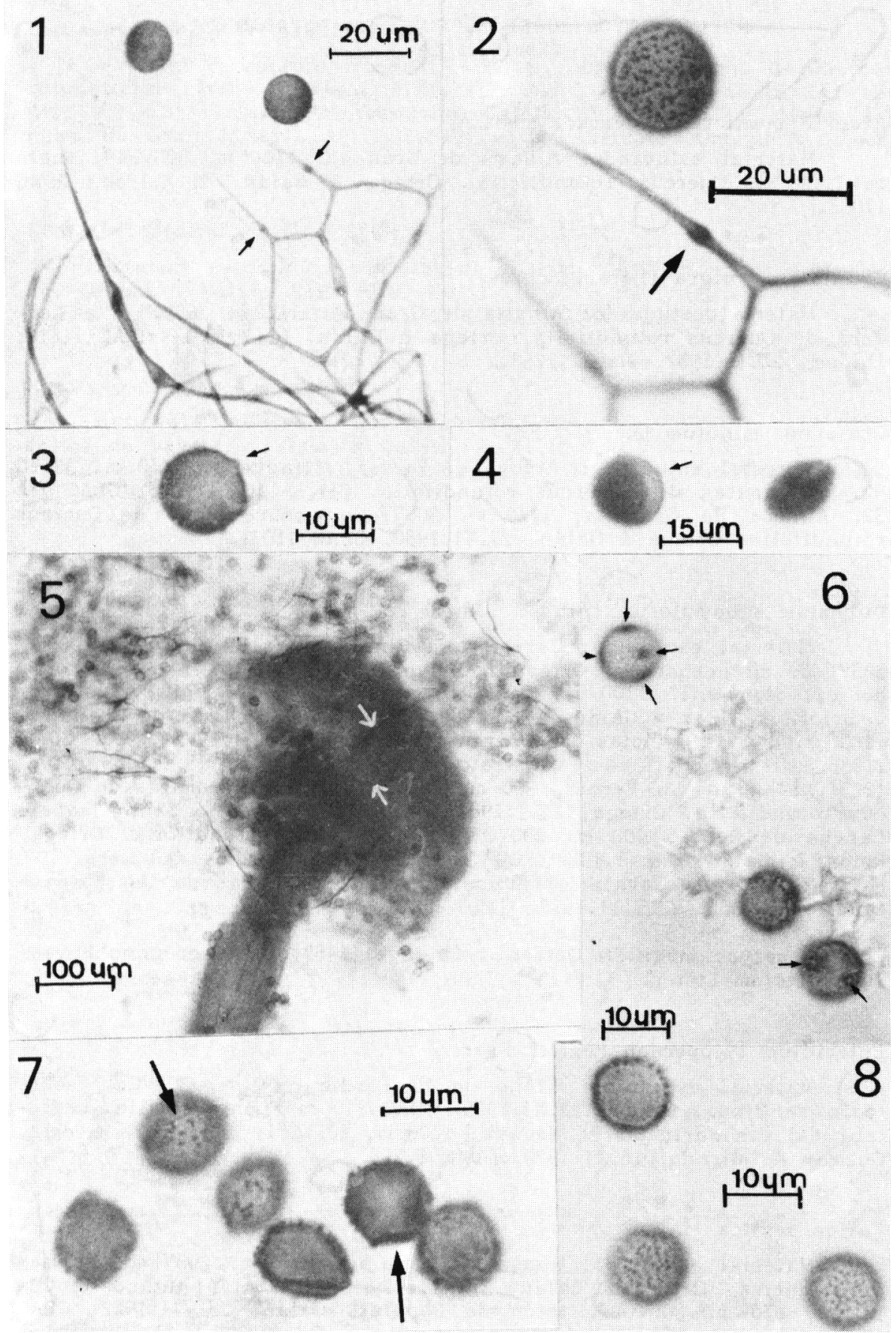

Lámina 1.- 1 y 2: detalle de los nódulos del capilicio y ornamentación esporal de Diderma trevelyanii. 3 y 4: detalles de la morfologia esporal de Didymium difforme. 5: filamentos del capilicio adosados a la pseudocolumnela en Didymium bahiense. 6: cristales de carbonato y grupos de verrugas más patentes en la ornamentación de la espora de Didymium bahiense. 7: esporas angulosas de Didymium trachysporum. 8: esporas de Physarum contextum . 
Arcyria pomiformis (Leers.) Rost.

Material estudiado: Alhama de Granada, $1140 \mathrm{~m}, 30$ SVF19, sobre ramitas de Quercus rotundifolia, Ortega \& Galán, 23.X.1980, GDAC 11023.

Comatrichia nigra (Pers.) Schroet.

Material estudiado: Alhama de Granada, $1140 \mathrm{~m}, 30 S V F 19$, en madera de Quercus rotundifolia, Ortega \& Galán, 6.IV.1981, GDAC 11416; Ibidem, 24.II.1982, GDAC 12253.

Craterium minutum (Leers.) Fr.

Material estudiado: Arroyo de Fardes, Alfaguara, $1440 \mathrm{~m}$, 30SVG 52, en ramitas de Quercus rotundifolia, Gil, 14.XII.1978, GDAC 110 32. Alhama de Granada, $1140 \mathrm{~m}, 30 \mathrm{SVF} 19$, sobre hojas de Quercus rotundifolia, Ortega \& Galán, 27.XI.1980, GDAC 11031.

Didymium squamulosum (Alb. \& Schw.) Fr.

Material estudiado: Carretera a Pinos del Valle, Güájares,1000m, 30SVF48, en herbáceas, Ortega \& Salvo,22.XI.1979,GDAC 12246. Los Cahorros, Monachil, $1100 \mathrm{~m}, 30$ SVG50, sobre hojas de Celtis australis y briófitos, Isac \& Galán, 17.V.1980, GDAC 11039. Alhama de Granada, $1140 \mathrm{~m}, 30$ SVF19, hojas de Quercus rotundifolia, Gil,19.XI.1980, GDAC 11038. Misma localidad y sustrato, Ortega \& Galán, 19.1.1982, GDAC 12251. Llano de la Perdiz, $900 \mathrm{~m}$, 30SVG41, hojas y ramitas de Quercus rotundifolia, Ortega, 15.I.1982, GDAC 12250. Cortijo Santa Isabel, Lancha de Cenes, $900 \mathrm{~m}, 30$ SVG51, hojas de Q. rotundifolia, Ortega, Prior \& Galán, 24.II.1982, GDAC 12248. Cercanias de Colomera, 700 m,30SVG32, sobre ramitas de Ulex parviflorus y hojas de Quercus rotundifolia, Ortega, 7.II.1979, GDAC 11061. rum bitectum Lister.

Observaciones: En Ortega \& Calonge (1981) fué dado como Physa-

Enteridium lycoperdon (Bull.) Farr

Material estudiado: Llano de la Perdiz, $900 \mathrm{~m}$, 30SVG41, sobre tocón de Pinus, Galán, 23.XI.1979, GDAC 11068. Fuente Agrilla, carretera del Seminario Sierra Nevada, $1400 \mathrm{~m}, 30 \mathrm{SVG} 61$, en madera muerta, Guzmán \& Guirado, 16.XI.1979, GDAC 12258.

Fuligo septica (L.) Wiggers

Material estudiado: Sierra de Cázulas, $1000 \mathrm{~m}, 30 \mathrm{SVF} 48$, en madera muerta, Ortega \& Galán, 16.XI.1979, GDAC 11041. Pantano de Cubillas, $650 \mathrm{~m}$, 30SVG32, tocón de Populus, Galán, 28.VII.1982, GDAC 12259 . 
Leocarpus fragilis (Dicks.) Rost.

Material estudiado: Monachil, $780 \mathrm{~m}$, 30SVG51, ramas de Quercus rotundifolia, Isac \& Galán, 3.XI.1979. GDAC 11048. Llano de la Perdiz, 900 m, 30SVG41, mismo sustrato, Galán, 23.XI.1979, GDAC 11046. Alhama de Granada, $1140 \mathrm{~m}, 30 \mathrm{SVF} 19$, mismo sustrato, Ortega \& Galán, 13.XI.1980, GDAC 11047.

Licogala epidendrum (L.) Fr.

Material estudiado: Soportújar, $1400 \mathrm{~m}, 30 \mathrm{SVF} 68$, madera de pino, Ortega \& Galán, 21.X.1979, GDAC 11052. Fuente de la Teja, Alfaguara, 1250 m, Gay \& Gallegos, 30.X.1979, GDAC 12257.

\section{Mucilago crustacea Wiggers}

Material estudiado: Los Cahorros, Monachil, $1000 \mathrm{~m}, 30$ SVG50, restos de vegetales, Isac \& Galán, 24.XI.1979, GDAC 11055. Cercanías de Colomera, $700 \mathrm{~m}$, 30SVG33, en ramas de Quercus rotundifolia,Galán, 24.III.1982, GDAC 12256.

Physarum bethellii Macbr.

Material estudiado: Arroyo de Fardes, Alfaguara, $1440 \mathrm{~m}, 30 \mathrm{SVG}$ 52, sobre restos vegetales, Ortega \& Galán, 12.XI.1980, GDAC 11458.

Physarum leucophaeum Fr.

Material estudiado: Alfaguara, $1400 \mathrm{~m}, 30 \mathrm{SVG} 52$, sobre piñas de Pinus pinaster, Ortega, 30.X.1980, GDAC 11256.

Physarum nutans Pers.

Material estudiado: Güéjar Sierra, $1400 \mathrm{~m}$, 30SVG61, entre la hojarasca de Quercus rotundifolia, Ortega \& Galán,17.X.1979, GDAC 11063. Dehesa del Camarate, $1430 \mathrm{~m}, 30$ SVG71, mismo sustrato, Ortega \& Galán, 20.XI.1980,GDAC 11064. Alhama de Granada, 1140 m, 30SVF19, mismo sustrato, Ortega, 12.XII.1980,GDAC 11426. Misma localidad y sustrato, Ortega \& Galán, 6.VI.1981, GDAC 11417.

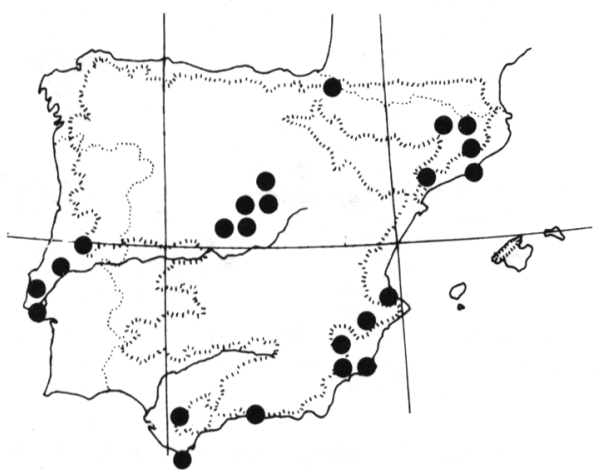

Mapa 1. Arcyria cinerea (Bull.) Pers.

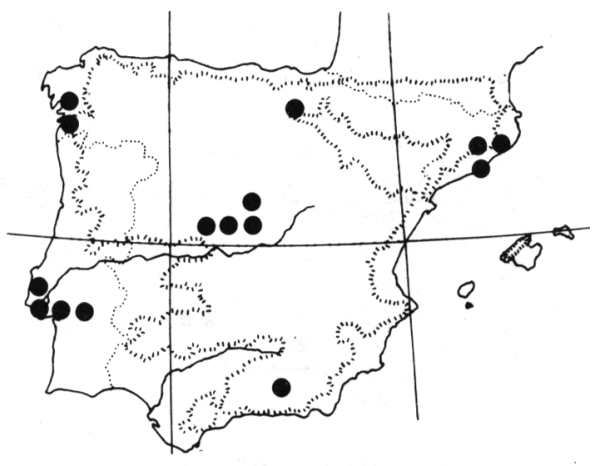

Mapa 2. Arcyria ferruginea Santer 


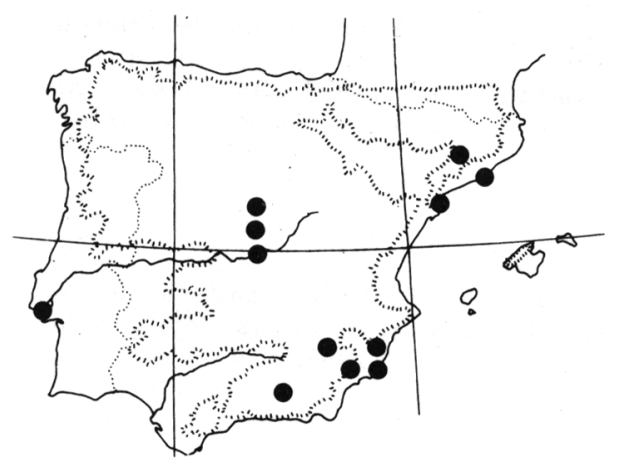

Mapa 3. Badhamia panicea (Fr.) Rost.

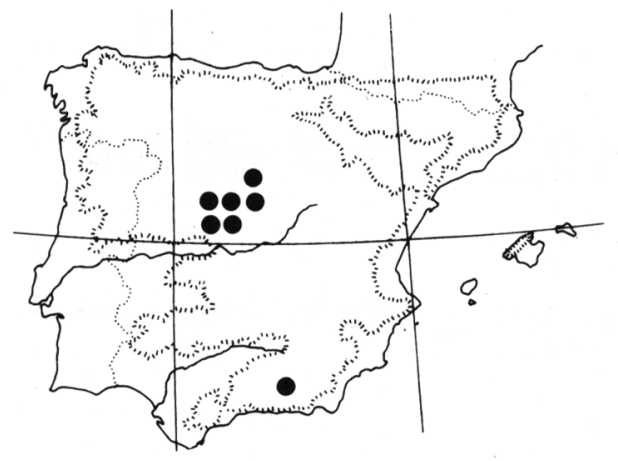

Mapa 5. Diderma travelyanii (Grev.) Fr.

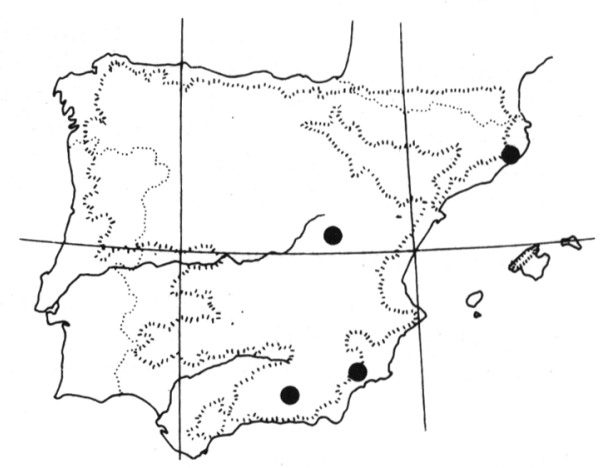

Mapa 4. Comatrichia alta Preusz

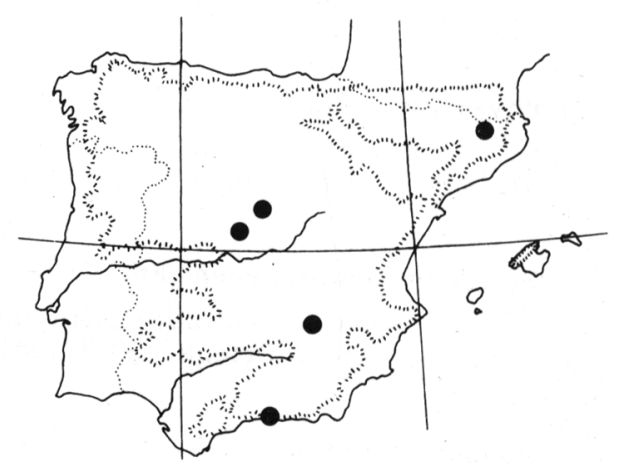

Mapa 6. Diderma umbilicatum Pers.

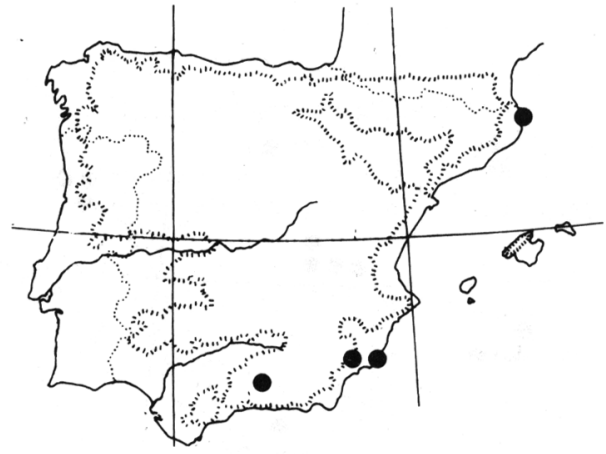

Mapa 7. Didymium bahiense Gottsberg. emend. Nann.-Brem.

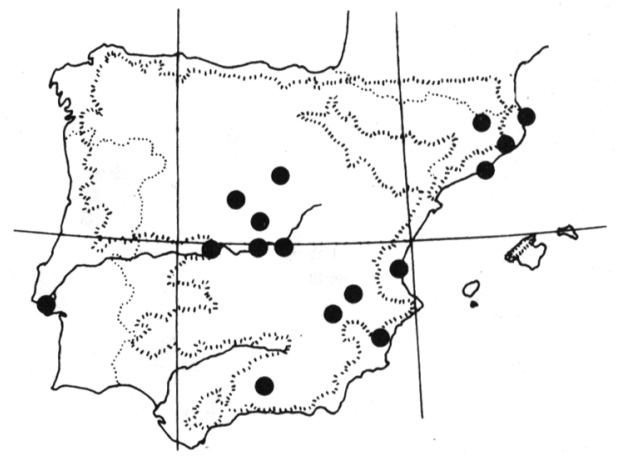

Mapa 8. Didymium difforme (Pers.) S.F. Gray 


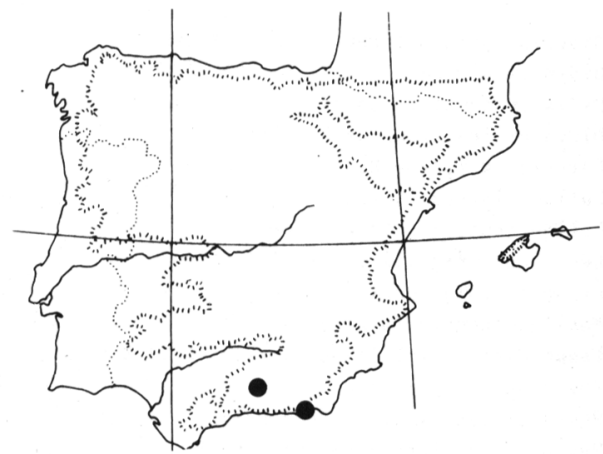

Mapa 9. Didymium trachysporum G. Lister

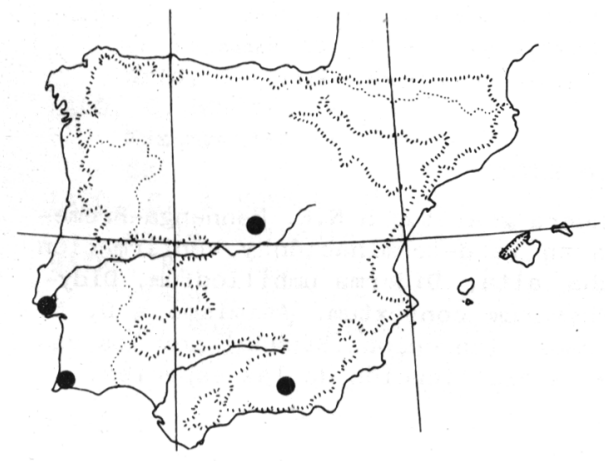

Mapa 11. Physarum contextum (Pers.) Pers. Mapa 12. Physarum vernum Somm.

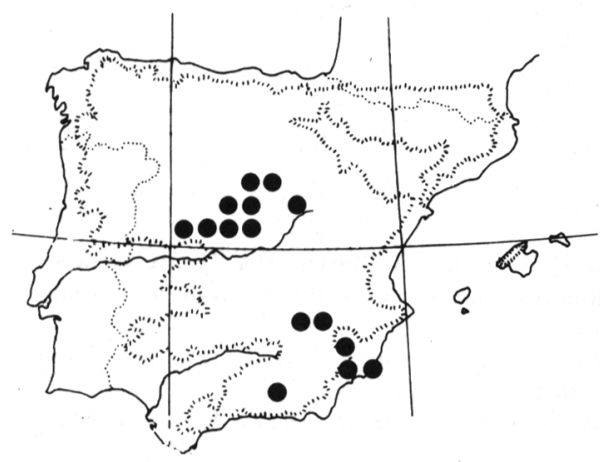

Mapa 13. Trichia contorta (Ditmar) Rost. var. contorta

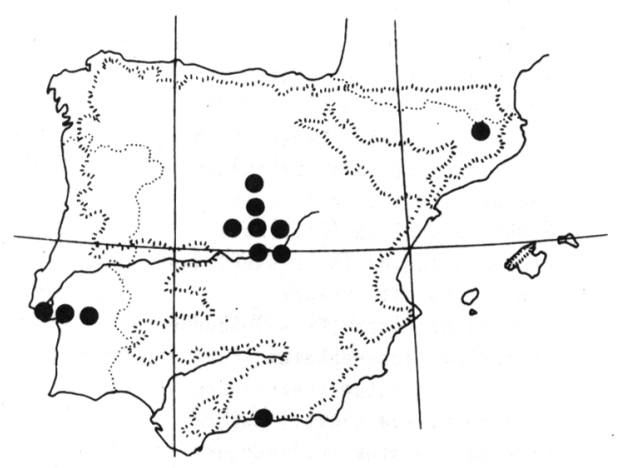

Mapa 10. Lamproderma scintillans (Berk. \& Br.) Morgan
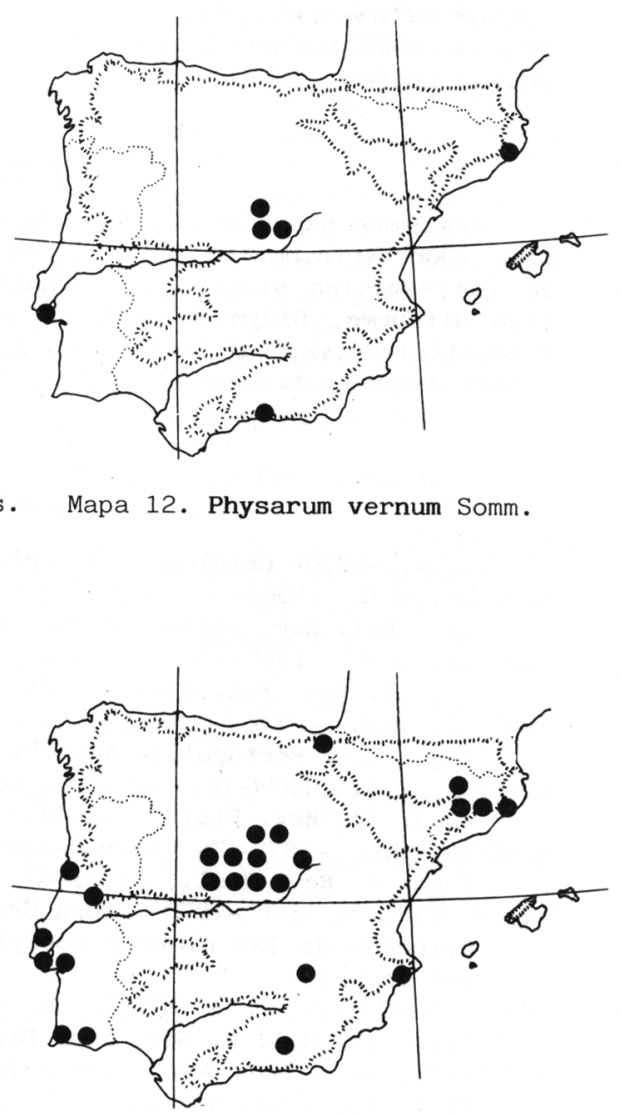

Mapa 14. Trichia varia (Pers.) Pers. 


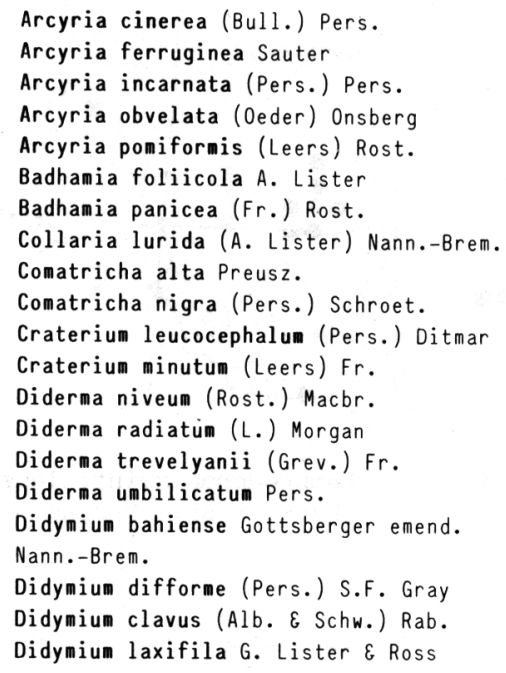

\author{
Didymium melanospermum (Pers.) Macbr. \\ Didymium nigripes (Lynk) $\mathrm{Fr}$. \\ Didymium squamulosum (Alb. \& Schw.) Fr. \\ Didymium trachysporum G. Lister \\ Enteridium lycoperdon (Bull.) Farr \\ Fuligo cinerea (Schw.) Morgan \\ Fuligo septica (L.) Wiggers \\ Leocarpus fragilis (Dicks.) Rost. \\ Lycogala epidendrum (L.) Fr. \\ Mucilago crustacea Wiggers \\ Lamproderma scintillans (Berk. \& Br.) Morgan \\ Physarum bethelii Macbr. \\ Physarum contextum (Pers.) Pers. \\ Physarum conglomeratum ( $F r$.) Rost. \\ Physarum leucophaeum $\mathrm{Fr}$. \\ Physarum nutans Pers. \\ Physarum vernum Somm. \\ Symphytocarpus impexus B. Ing. \& Nann.-Brem. \\ Irichia contorta (Ditmar) Rost. \\ Trichia decipiens (Pers.) Macbr. \\ Irichia varia (Pers.) Pers.
}

\section{AGRADECIMIENTOS}

Queremos expresar nuestra más sincera gratitud a N.E. Nannenga-Bremekamp (Doorwerth, Holanda) por la ayuda en la determinación y confirmación de las especies siguientes: Comatricha alta, Diderma umbilicatum, Didymium difforme, Didymium bahiense y Physarum contextum. Asimismo a D. N. Mitchell (Sussex, Inglaterra) y a E. Cracia (Barcelona,España) por los valiosos datos gentilmente cedidos sobre la distribución de las especies.

\section{BIBLIOGRAFIA}

A.E.P.N.A. -1976- Catálogo micológico de Alava. Diputación Foral de Alava.

ALMEIDA, M.G. -1964- Contribuçao para o estudo dos Myxomycetes de Portugal. Bol. Soc. Portug. Cienc. Nat., 10:172-185.

ALMEIDA, M.G. -1966- Contribuçao para o estudo dos Myxomycetes de Portugal II. Rev. Fac. Cienc. Lisboa, 14 (1): 5-12.

ALMEIDA, M.G. -1972- Contribuçao para o estudo dos Myxomycetes de Portutugal III. Portugaliae Acta Biol., 11 (3-4): 58-273.

ALMEIDA, M.G. -1974-76- Contribuçao para o estudo dos Myxomycetes de Portugal IV. Rev. Biol., 10(1-4):113-125.

ALMEIDA, M.G. -1977-79- Contribuçao para o estudo dos Myxomycetes de Portugal V. Rev. Biol., $11(1-4)$ : 79-90.

AMO Y MORA, M. del -1870- Flora Criptogámica de la Península Ibérica;descripción de las plantas Acotyledóneas que crecen en España y Portugal. Granada.

BENITO MARTINEZ, J. \& GUINEA, E. -1931- Nueva aportación a la micoflora española. Bol. R. Soc. Esp. Hist. Nat., 31:211-220.

CABALlero, A. -1928- Adiciones a la micoflora española. Bol. R. Soc. Esp. Hist. Nat., 28: 421-430.

CHECA, J.,LADO, C. \& MORENO, G. -1982- Notas sobre los Myxomycetes de los hayedos del macizo de Ayllón. Bol. Soc. Micol. Cast., 7:7-18.

CUATRECASAS, J. -1925- Algunos datos para la flora mixomicética de Cataluña. Bull. Inst. Catalana Hist. Nat., 5: 92-95.

FONT-QUER, P. -1923- Notes criptogámiques. Bull. Inst. Catalana Hist. Nat., 3: 31-32. 
GARCIA MANJON, J.L. -1978- Hongos que fructifican sobre el género Pinus L. en España. Tesina Inéd. Fac. Biología, Univ. Complutense. Madrid.

GRACIA, E. -1977- Contribución a la flora de Mixomicetos de Cataluña. Mediterranea, 2:79-87.

GRACIA, E. \& LLIMONA, X. -1980- Contribución al conocimiento de la flora y distribución de los mixomicetes en el Mediterráneo Occidental. III. Sureste de España: Murcia. Anal. Univ. Murcia Ciencias, 34 (1-4):321 .

GRACIA, E., HONRUBIA, M. \& LLIMONA, X. -1981- Aportación al conocimiento de los hongos del S.E. de España.II.Mixomicetes de la provincia de Albacete. Anal. Univ. Murcia Ciencias, 37(1-4): 63-79.

GRACIA, E. -1981- Estudio sobre la Flora, Fitosociología, Ecología y Corología de los Myxomycetes de España. Tesis Doc. inéd. Univ. de Barcelona.

KOWALSKI, D.T. -1970- The species of Lamproderma. Mycol., 62 (4): 621-672.

LADO, C. \& MORENO, G. -1976-Contribución al estudio de los Myxomycetes en España Peninsular. I. Anal. Inst. Bot. Cavanilles, 33:111-124.

LADO, C. \& MORENO, G. -1978-Contribución al estudio de los Myxomycetes en España Peninsular. II. Anal. Inst. Bot. Cavanilles, 34 (2): 401415 .

LADO, C. \& MORENO, G. -1980-Contribución al estudio de los Myxomycetes en España Peninsular. III. Provincia de Madrid. Anal. Jard. Bot. Madrid, 37(1): 5-30.

LADO, C., MORENO, G., ORTEGA, A. \& CALONGE, F. de -1980- Estudios sobre Myxomycetes.IV. Provincia de Granada. Bol. Soc. Micol. Cast., 5: 5568.

LADO, C. \& MORENO, G. -1981-Contribución al estudio de los Myxomycetes en la Península Ibérica.IV. País Vasco. Munibe, 33 (1-2): 63-78.

MAIRE, R., CODINA, J. \& FONT-QUER, P. -1933- Fungi Catalaunici. Contributions à l'étude de la Flore Mycologique de la Catalogne. Treballs del Museu de Ciencias Naturals de Barcelona, 15 (2): 1-120.

MAS Y GUINDAL, J. -1945- Catálogo provisional de la Flora Criptogámica de la provincia de Madrid. An. R. Acad. Farm., 11: 407-474.

MARTIN, G.W. \& ALEXOPOULOS, C.J. -1969- The Myxomycetes. Univ. of Iowa Press.

NANNENGA-BREMEKAMP, N.E. -1968- Notes on Myxomycetes XVI. Remarks on some species of Diderma. Proc. K. Ned. Akad. Wet. ser. C, 71 (2): 189-200.

NANNENGA-BREMEKAMP, N.E. -1972- Notes on Myxomycetes XVIII. A new Didymium and some comments on the Didymium species with long-stalked sporangia. Proc. K. Ned. Akad. Wet. ser C, 75 (4): 352-363.

NANNENGA-BREMENKAMP, N.E. -1974- De Nederlandse Myxomycete. K. Nederl. Natuurhist. ver. Zutphen.

RIVAS-MARTINEZ, S. -1981- Les étages bioclimatiques de la végétation de de la Péninsule Ibérique. Anal. Inst. Bot. Cavanilles, 37 (2): 251268 .

TORREND, C. -1908- Cataloge raissonné des Myxomycètes de Portugal. Bull. Soc. Port. Sc. Nat., 2(1-2): 55-73.

URRIES, M.J. -1953- Hongos microscópicos de Navarra (segunda nota). Anal. Inst. Bot. Cavanilles, 11: 153-160.

Dirección de los autores: R. GALAN y C. LADO, Departamento de Botánica, Universidad de Alcalá de Henares, Madrid. A. ORTEGA, Departamento de Botánica, Facultad de Ciencias, Universidad de Granada. 